\title{
Study of Alpha Particle Impact Double Ionization Cross Sections of Cu Atom
}

\author{
S. P. Gupta, K. Yadav, R. Ghimire, R. Khanal and L. K. Jha
}

Journal of Nepal Physical Society

Volume 7, Issue 4, December 2021

ISSN: 2392-473X (Print), 2738-9537 (Online)

\section{Editors:}

Dr. Binod Adhikari

Dr. Bhawani Joshi

Dr. Manoj Kumar Yadav

Dr. Krishna Rai

Dr. Rajendra Prasad Adhikari

Mr. Kiran Pudasainee

JNPS, 7 (4), 43-51 (2021)

DOI: http://doi.org/10.3126/jnphyssoc.v7i4.42930
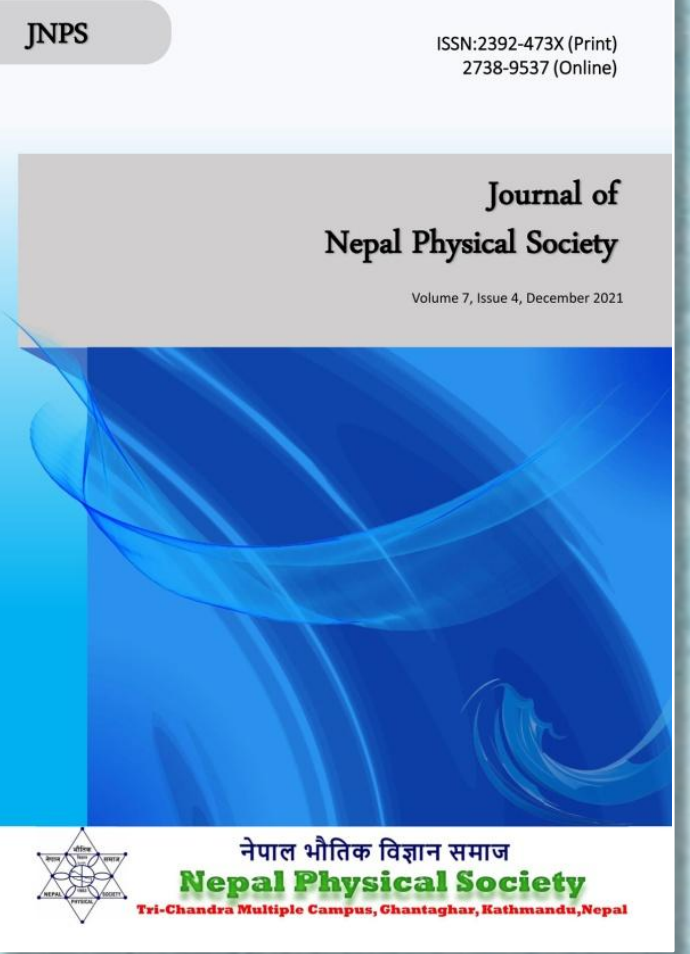

Published by:

Nepal Physical Society

P.O. Box: 2934

Tri-Chandra Campus

Kathmandu, Nepal

Email: nps.editor@gmail.com 


\title{
Study of Alpha Particle Impact Double Ionization Cross Sections of $\mathrm{Cu}$ Atom
}

\author{
S. P. Gupta ${ }^{1}$, K. Yadav ${ }^{1}$, R. Ghimire ${ }^{1}$, R. Khanal ${ }^{2}$ and L. K. Jha ${ }^{3}$ \\ ${ }^{1}$ Patan Multiple Campus, Tribhuvan University, Lalitpur, Kathmandu, Nepal \\ ${ }^{2}$ Central Department of Physics, Kirtipur, Tribhuvan University, Nepal \\ ${ }^{3}$ Department of Physics, B R A Bihar University, Muzaffarpur-842001, Bihar, India \\ "Corresponding Email: suresh.gupta@pmc.edu.np
}

Received: 0 13th November, 2021; Revised: 18th December, 2021; Accepted: 29th December, 2021

\begin{abstract}
Semi-classical binary encounter approximation has been used for the study of ionization cross sections of $\mathrm{Cu}$ atom at ground state. Projectile, alpha particle, with energy varying from threshold to $360 \mathrm{keV} / \mathrm{amu}$ are considered for direct double ionizations cross section. Out of the two path ways of ionization we have ruled out the indirect process (like excitation-auto ionization, Auger effect) of double ionization. We considered direct - ionization of outer shells of $\mathrm{Cu}$ atom. Vriens accurate expression of cross section for energy transfer $\Delta E\left(\sigma_{\Delta E}\right)$ and Hertree-Fock velosity distribution function for the target electrons are used. The theoretical value of double ionization cross sections at impact energy $300 \mathrm{keV} / \mathrm{amu}$ is $0.98 \times 10^{-16} \mathrm{~cm}^{2}$ which is same as experimental values at that impact energy. Above energy of $300 \mathrm{keV} / \mathrm{amu}$, the calculated results underestimates the experimental data and at $360 \mathrm{keV} / \mathrm{amu}$ their magnitude are $0.77 \times 10^{-16} \mathrm{~cm}^{2}$ and $1.0 \times 10^{-16} \mathrm{~cm}^{2}$ respectively. Above impact energy of $75 \mathrm{keV} / \mathrm{amu}$, all the theoretical results have ratio factor within 2. Out of the total number of theoretical data $75 \%$ are under valid range of ratio factor 2 and among the valid range $41 \%$ have ratio factor below 1.325 and hence calculated results of double ionization cross section lie in the acceptable range. The linear correlation coefficient (R square) and standard deviation (SD) of linear fit are 0.6984 and 0.7883 respectively. In low energy range the theoretical results are more apart from corresponding experimental values and possess relatively more error compared to intermediate and higher energy region. Calculated results are in satisfactory agreement with experiment in intermediate and high energy range.
\end{abstract}

Keywords: Binary encounter approximation, Double ionization cross section, Alpha particle, Hartree-Fock velocity distribution, Vrien's expression of accurate cross section $\left(\sigma_{\Delta E}\right)$.

\section{INTRODUCTION}

Many theoretical and experimental investigations have been devoted to gaining a thorough knowledge of the electronic dynamics and structure of atoms and molecules. A common technique for extracting information from such small entities is to perturb the system. The perturbation must be strong enough to compete with the strong Coulomb interactions inside atoms. The use of intense laser pulses in strong-field physics and particle impact in atomic collision physics are two examples of typical procedures. The perturbation must be powerful enough to overcome the strong Coulomb interactions that exist within atoms. The fundamental mechanisms that occur during atom ionization are divided into two categories: direct ionization processes, in which ionization occurs immediately after impact, and indirect ionization processes, such as excitation auto-ionization, in which ionization occurs after the hit. The energy areas that interact with atomic electrons are characterized as [1].

(i) The low-energy region, where only the outer shell is involved in ionization processes. 
(ii) The intermediate-energy region, where the inner shell can also be involved in ionization processes.

(iii) The high-energy region, where very few atoms are ionized because the interaction times are too brief.

Collisions between target atoms and heavy particles such as $\mathrm{H}+$ and $\mathrm{He} 2+$ can result in pure ionization, excitation, excitation-auto ionization, electron capture, charge transfer, and transfer ionization. Continuous data on respective process ionization cross sections is critical in a variety of fields of study, including fusion reactor modeling, Titan's upper atmosphere, comet plasma processes, planetary atmospheres, and biological applications [2-4]. For both therapeutic and palliative reasons, radiotherapy is an effective and frequently used treatment for cancer patients. Ionization and fragmentation of atoms and molecules have been employed in cancer therapy. Some suitable ionizing agents cause water molecules in the human body to fragment, resulting in the creation of numerous reactive radicals that can cause local biological damage near the tumor and aid in treatment. The damage is caused by the deposition of radiation energy into the DNA. Intermediate species such as ions, radicals, excited molecules, and free electrons are produced by the energy deposition, and these species then interact with DNA. A projectile particle, such as a proton or helium, deposits a huge amount of energy in a volume of a few micrometers or even nanometers, causing substantial damage to the microscope structure and DNA cell death $[5,6]$. Second, electron collisions in atmospheres determine the abundance of some of their minor constituents, which can or may play a large role in energy transmission. Radiative emissions are important for remote sensing and can be caused by electron impact excitation. Both scenarios necessitate computational modeling, which necessitates accurate and comprehensive atomic and molecular physics data of cross sections and chemical reaction rates. This type of research is applied in a variety of physics issues. The mechanism of the'sun-weather' correlation, modelling of NO production in the upper atmosphere [7], calculations of heating rates in the atmosphere of Titan, simulation of infrared emissions from NO in an aurora [8], modelling of the Earth's green nightglow [9], are just a few of the physics topics that are currently of interest. Their needs precise data of atomic and molecular cross sections and chemical reaction rates. This type of research is applied in a variety of physics research. Finally, in plasma physics, where electrons and ions react with one other, and plasma-eaching of microchips, the study of collision dynamics and exact data of cross sections are used. Plasmaassisted procedures are used to treat surfaces, materials, and some electronics in an indirect manner to achieve specific features [10]. Physical plasma has become increasingly popular for medicinal purposes, and it continues to be a cuttingedge and developing subject. It can be used to provide therapeutic effects in either the human or animal body [11, 12] including sterilisation, disinfection, and decontamination for medicinal uses [13]. For simulating the track structure of radiation, cross section data for ion and electron scattering in gases is necessary. The accuracy of this data is critical for the precise calculation of transport parameters and ionization yields in detector development, space research, and radiation dosimetry applications. These figures are derived from observations or theoretical computations. When heavy charged particles $(\mathrm{H}+$ or $\mathrm{He} 2+)$ collide with target atoms, they can produce pure single and double atoms ionization. Multiple ionization is a multi-electron process in which both direct and indirect ionization play a role in the final charge state. Because of the intricate nature of the fourbody Coulomb potential, where correlation effects are the most important, direct double ionization is garnering a lot of attention from researchers. Pindzola et al. [14, 15] have successfully used a time-dependent close coupling technique to evaluate systems with more than two electrons. Montanan et al. [16] used a quantum mechanical model of a continuum distorted wave ekonal starting state to explore repeated ionization of $\mathrm{Ar}$ caused by the impact of an alpha particle (CDWEIS). At high energies, the theoretical results studied were highly consistent with practical data. Despite these achievements, the mathematical formulation for the calculation of single and multiple ionization cross sections of heavy atoms. These findings, however, only apply to systems with one or two electrons outside the core, and thus are completely useless for more complicated systems due to extremely precise calculations. As a result, there are no sophisticated estimations of the total double ionization cross sections of multiple electron atoms by ion impact in the literature. The Binary Encounter Approximation (BEA) has been successfully used in the past in calculations of charged particle impact single and double ionization cross sections for several atoms, as well 
as calculations of single and multiple ionization cross sections of heavy atoms under quantal approximations.

In the beginning theoretical studies on application of the classical binary encounter approximation (BEA) were carried out by Gryzinski [17] through series of research papers.

Gryzinski reasonably considered two processes in a double Binary Encounter Model to describe double ionization. In the first process, the two electrons may be ejected from the system by two successive interactions of the incident particle with the target electrons. Next, the incident particle may knock out only one target electron and the second electron is removed by the first ejected electron. The corresponding cross sections are denoted by $Q_{s c}^{i i}$ (scattered part) and $Q_{e j}^{i i}$ (ejected part) respectively. The results of double ionization cross sections based on the modified model including contributions of indirect physical processes were found to be in close agreement with the experimental data $[18,19]$. In these calculations Hatree-Fock (HF) and hydrogenic velocity distributions were used while considering ejection of the first and the second target electron respectively. Later on, Jha and Roy $[20,21]$ used HF velocity distribution while considering the ejection of both electrons of the target in calculations of direct double ionization cross sections. In case of heavy charged particle the binary encounter calculations of double ionization cross sections of atoms are scarce in literature. Kumar and Roy [22] pointed out errors and obscurities in Gryzinski's theory for calculations of the above mentioned processes and modified the mathematical framework suitably incorporating the necessary corrections of using the accurate expression of $\sigma_{\Delta E}$ (cross section for energy transfer $\Delta \mathrm{E}$ ) as given by Vriens [23]. They calculated proton impact double ionization cross sections of noble gases [24, 25] which were found to be in satisfactory agreement with the experimental observations. Later on, Singh et al [26] have used HF velocity distribution function for considering the ejection of both the electrons respectively in case of $\mathrm{H}+$ and $\mathrm{He}^{2+}$ impact double ionization of $\mathrm{Mg}$ and found satisfactory agreement with the experiment. Gryzinski and Kune [27] conceived an idea of double binary encounter for double ionization. According to Gryzinski and Kune the two active target electrons may ejected either by two successive encounters with the incoming charged particle or the first one ejected electron having sufficient energy ejects second active target electron. The former and later processes of ionization are usually denoted by $Q_{s c}^{i i}$ (called scattered part) and $Q_{e j}^{i i}$ (called ejected part) respectively. The total direct double ionization cross section (DDICS) for the impact of charged particle (ignoring Auger effect) can be given as $Q_{D}^{i i}=Q_{s c}^{i i}+Q_{e j}^{i i}$. Keeping the above mentioned facts in view, we have carried out computational calculation of alpha particle impact double ionization cross section of $\mathrm{Cu}$ under modified semiclassical BEA. This work will enable us to analyze direct double ionization cross sections.

\section{THEORETICAL DETAILS AND METHODS OF CALCULATION}

Thomson first used the binary encounter theory for calculating cross section for ionization of atom by electrons. According to Thomson (1912) consider a situation of collision where the energy transfer in Coulomb collision between a particle of mass $m_{1}$ and charge $Z_{1} e$ with initial kinetic energy $E_{1}$ and a particle of mass $m_{2}$ and $Z_{2} e$ with initial kinetic energy $E_{2}=0$ (rest). In the case of binary encounter theory it has been assumed that during the period of interaction between projectile and an orbital electron the other atomic electrons and the nucleus play no role. The Thomson's energy $\operatorname{transfer}(\varepsilon)$ ionization cross section for electronelectron collision is [28]

$$
\frac{d Q(\varepsilon)}{d \varepsilon}=\pi \frac{e^{4} N}{E_{1}}\left[\frac{1}{U}-\frac{1}{E_{1}}\right]
$$

For ionization $U \leq \varepsilon \leq E_{1}$; where $U$ is ionization potential energy.

Thomas and William (1927) modified the formulation for more general case where $E_{2} \neq 0$ (considered symmetrical distribution of velocity of target electrons), $\left.\left.m_{1}\right\rangle\right\rangle m_{2}$ and $Z_{1} \neq Z_{2}$ which is relevant to proton and alpha particle -atom collision. Energy transfer ionization cross section for this case has been given as [28]

$$
\frac{d Q(\varepsilon)}{d \varepsilon}=\pi \frac{e^{4} Z_{1}^{2} Z_{2}^{2} m_{1}}{m_{2} E_{1}}\left[\frac{1}{\varepsilon^{2}}+\frac{4 E_{2}}{3 \varepsilon^{3}{ }_{1}}\right]
$$

Gryziniski obtained classical relations for Coulomb collision of two moving charged particles and 
applied them for theoretical studies of a varity of charge particle- atom collision processes. He assumed to have a velocity distribution or a single average velocity. The excitation cross section is for a transition to a principal quantum number not to the individual sublevels. Gryzinski assumed s-s transitions only take place during exchange process.
Gryzinski improved the Thomson's equation by assuming a continuous velocity distribution for the atomic electrons leading to the following expression of cross section [29]

$$
\sigma=\sum 4 \pi a_{0}^{2} N_{n}\left(\frac{E_{i}^{H}}{E_{\text {in }}}\right)^{2} f(u)
$$

where,

$$
f(u)=\frac{1}{u}\left[\frac{(u-1)}{(u+1)}\right]^{3 / 2}\left\{1+\frac{3}{2}\left(1-\frac{1}{2 u}\right) \ln \left(2.7+(u-1)^{1 / 2}\right)\right\}
$$

Here $a_{0}$ is the Bohr's radious, $N_{n}$ and $E_{i n}$ are number of electrons and ionization potential of the $n^{\text {th }}$ sub shell, $E_{i}^{H}$ is the ionization potential of the theory requires the incorporation transfer of the hydrogen atom and $u=E / E_{\text {in }}$, where $E$ is incident energy. Further improvement in the theory requires the incorporation of the quantum factors such as exchange and interference effects. Vrien's modified the Gryzinski's theory and gave a set of quantum mechanical formula in terms of momentum transfer as a variable. He incorporated symmetrical properties that includes exchange and interference effects and finally obtained differential cross section for momentum and energy transfer.

We carry out theoretical calculations of alpha particle $\left(\mathrm{He}^{2+}\right)$ impact double ionization cross sections of $\mathrm{Cu}$ atom using the modified BEA. The

theoretical approach used in BEA is based on independent particle model (IPM). The model is based on the hypothesis that the probability of ionizations is directly related to the energy deposited by the projectile on the target. The energy deposited is statistically distributed among all atomic electrons and one or more of which eventually auto ionize to the final state. Here, an accurate expression of $\sigma_{\Delta E}$ (cross section for energy $\operatorname{transfer} \Delta E$ ) for proton impact given by Vriens [23] and quantum mechanical Hartree -Fock velocity distribution functions have been used to calculate the ionization cross sections of the atom.

As discussed the total direct double ionization cross section for the impact of charged particle (ignoring Auger effect) can be given as

$$
Q_{D}^{i i}=Q_{s c}^{i i}+Q_{e j}^{i i}
$$

where

$$
Q_{s c}^{i i}=\frac{n_{e}\left(n_{e}-1\right)}{4 \pi \bar{r}^{2}} \times \int_{u_{i}+u_{i \bar{i}}}^{\Delta E_{\max }} \sigma_{\Delta E}\left(E_{q}\right)\left(\int_{u_{i \bar{i}}}^{\Delta E_{\max }} \sigma_{\Delta E}\left(E_{q}-\Delta E\right) d\left(\Delta E^{\prime}\right)\right) d(\Delta E)
$$

and

$$
Q_{e j}^{i i}=\frac{n_{e}\left(n_{e}-1\right)}{4 \pi \bar{r}^{2}} \times \int_{u_{i}+u_{i \bar{i}}}^{\Delta E_{\max }} \sigma_{\Delta E}\left(E_{q}\right)\left(\int_{u_{\bar{i}}}^{\Delta E-u_{i}} \sigma_{\Delta E^{\prime}}(\Delta E) d\left(\Delta E^{\prime}\right)\right) d(\Delta E)
$$

In the present calculations, an accurate expressions of differential cross section $\sigma_{\Delta E}$ (cross section for energy transfer $\Delta E$ ) under different limits of energy transfer is used [25] and given as

$$
\sigma_{\Delta E} d(\Delta E)=\left\{\begin{array}{rr}
A d(\Delta E) ; & \Delta E \leq 4 s u(s-t) \\
B d(\Delta E) ; & 4 s u(s-t) \leq \Delta E \leq 4 \operatorname{su}(s+t) \\
0 ; & \Delta E>4 \operatorname{su}(s+t)
\end{array}\right\}
$$

where $A=\frac{4}{s^{2} u}\left(\frac{1}{(\Delta E)^{2}}+\frac{4 t^{2} u}{3(\Delta E)^{3}}\right)$ and $B=\frac{2}{3 t(\Delta E)^{3}}\left(8 s-\frac{\left|\left(\Delta E+t^{2} u\right)^{1 / 2}-t u^{1 / 2}\right|^{3}}{s^{2} u^{3 / 2}}\right)$. 
All the symbols appeared in above expressions have been defined by Gryzinski. In the above expressions $\Delta E, \Delta E^{\prime}$ represent transfer of energy during first and second collision with the two active electrons of the target, $E_{q}$ is the energy of the projectile.

Following McDowell [30], the kinetic energies of projectile $\left(m_{1} s^{2} u\right)$ and orbital electron $\left(t^{2} u\right)$ are expressed in terms of dimensionless variables $s$ and $t$ defied as $s^{2}=v_{1}^{2} / v_{0}^{2}$ and $t^{2}=v_{2}^{2} / v_{0}^{2}$, where $v_{1}$ and $v_{2}$ are the velocities of incident particle and target orbiting electron in atomic units respectively and $v_{0}$ is root mean square velocity of orbital electrons. The ionization potential energy of bound electron $u$ is defined as $u=v_{0}^{2}$. Atomic electrons are taken to have a momentum distribution and can be given by Fourier transformation of the Hartree Fock density distribution that includes quantummechanical velocity distribution for the bound electrons.

The expressions of the scattered part of the direct double ionization cross sections (DDICS) for positive and negative values of $(s-1 / 4 s)$ are given as

$Q_{s c}^{i i}=\frac{n_{e}\left(n_{e}-1\right) Z^{2}}{4 \pi \bar{r}^{2}} \times\left(\int_{t=0}^{s-\frac{1}{4 s}}\left\{\int_{u_{i}}^{4 s u_{i}(s-t)} A \alpha d(\Delta E)+\int_{4 s u_{i}(s-t)}^{4 s u_{i}(s+t)} B \alpha d(\Delta E)\right\} f(t) u_{i}^{1 / 2} d t+\int_{t=s-\frac{1}{4 s}}^{\infty} \int_{u_{i}}^{4 s u_{i}(s+t)} B \alpha f(t) u_{i}^{1 / 2} d(\Delta E) d t\right)\left(\pi a_{0}^{2}\right)$

and

$Q_{s c}^{i i}=\frac{n_{e}\left(n_{e}-1\right) Z^{2}}{4 \pi \bar{r}^{2}} \times\left(\int_{t=\frac{1}{4 s}-s}^{\infty} \int_{u_{i}}^{4 s u_{i}(s+t)} B \alpha f(t) u_{i}^{1 / 2} d(\Delta E) d t\right)\left(\pi a_{0}^{2}\right)$

In the above expressions $\alpha=\int_{0}^{\infty} Q_{i}\left(s^{\prime}, t\right) f^{\prime}(t) u_{i i}^{1 / 2} d t\left(\pi a_{0}^{2}\right)$

Similarly, the expressions of ejected part for positive and negative values of $s-\frac{\left(1+u_{i i} / u_{i}\right)}{4 s}$ are

$Q_{e j}^{i i}=\frac{n_{e}\left(n_{e}-1\right) Z^{2}}{4 \pi \bar{r}^{2}} \times\left(\int_{t=0}^{s-\left(1+\frac{\left.u_{i}\right)}{u_{i}} / 4 s\right.}\left\{\int_{u_{i}+u_{i}}^{4 s u_{i}(s-t)} A \alpha^{\prime}(\Delta E)+\int_{4 s u_{i}(s-t)}^{4 s u_{i}(s+t)} B \alpha^{\prime} d(\Delta E)\right\} f(t) u_{i}^{1 / 2} d t+\int_{t=s-\left(1+\frac{u_{i}}{u_{i}}\right) / 4 s}^{\infty} \int_{u_{i}+u_{i}}^{4 s u_{i}(s+t)} B \alpha^{\prime} f(t) u_{i}^{1 / 2} d(\Delta E) d t\right)\left(\pi a_{0}^{2}\right)$

and

$Q_{e j}^{i i}=\frac{n_{e}\left(n_{e}-1\right) Z^{2}}{4 \pi \bar{r}^{2}} \times\left(\int_{t=\left(1+\frac{u_{i}}{u_{i}}\right) / 4 s-s}^{\infty} \int_{u_{i}+u_{i}}^{4 s u_{i}(s+t)} B \alpha^{\prime} f(t) u_{i}^{1 / 2} d(\Delta E) d t\right)\left(\pi a_{0}^{2}\right)$

Where $u_{i}$ and $u_{i i}$ stand for first and second ionization potentials of target atom and $s^{\prime}$ is dimensionless variable defined as ratio of energy associated to alpha particle after first collision to second ionization potential (IP) and defined as $s^{\prime 2}=\frac{E_{q}-\Delta E}{7344 u_{i i}}$. The integral appearing in $Q_{s c}^{i i}$ and $Q_{e j}^{i i}$ have been evaluated numerically. In the above equations the functions $f(t)$ and $f^{\prime}(t)$ are HarteeFock momentum distributions functions corresponding to the first and the second ejected electrons respectively. The Hartree-Fock momentum distribution functions can be constructed from analytical Hartree-Fock radial function, normalization constant and corresponding spherical harmonics as discussed below [equations $(9-13)]$. The Hartree-Fock momentum distribution function $f(t)$ has been defined as,

$$
f(t)=4 \pi t^{2} u \rho_{n l}\left(u^{1 / 2} t\right)
$$

where $\rho_{n l}=\frac{1}{2 l+1} \sum_{-1}^{+1}\left|\psi_{n l m}(x)\right|^{2}$

and 
$\psi_{n l m}(r)=\frac{1}{(2 \pi)^{\frac{1}{2}}} \int \phi_{n l m}(r) e^{i k . r} d r$ is the Fourier

transform of the one electron orbital.

The complete wave function is given by

$\phi_{n l m}(r)=N_{n l} R_{n l}(r) Y_{l m}(\Omega)$

where $N_{n l}$ and $R_{n l}(r)$ are the normalization constant and analytical Hartree-Fock radial function, respectively. The empirical relations for $N_{n l} \& R_{n l}(r)$ are

$N_{n l}=[(2 n) !]^{-\frac{1}{2}}(2 \xi)^{n+\frac{1}{2}}$

and

$R_{n l}=r^{n-1} e^{-\xi r}$

Here zeta $(\xi)$ is orbital exponent of basis function. The spherical harmonic $Y_{l m}(\Omega)$ have different forms depending upon the value of orbital and magnetic quantum numbers $l$ and $m$ respectively. It is well known that velocity of orbital electrons increases with the decrease in shell number and hence velocity of inner shell electrons have relativistic in nature. The above expressions both $Q_{s c}^{i i}$ and $Q_{e j}^{i i}$ found to be dependent on square of the charge of incoming particle $\left(Z^{2}\right)$.Variens observed that the two double binary encounter process are linked quantum mechanically as first and second order approximations and the cross sections scales as $Z^{4}$.In the case of proton impact $(Z=1)$ as discussed by Singh et al. [26] and others presence of $Z^{4}$ does not alter the result of cross section compared to the presence of $Z^{2}$. However in the case of alpha particle impact ( $Z=2$ ), scaling of $Z^{4}$ gives much dominant contribution and produce adverse effect to the results. The adverse results suggest that both the scattered and ejected part of double ionization cross sections processes in the first and second Born approximation does not seems to be applicable. Therefor here we only consider $Z^{2}$ dependence of double ionization of $\mathrm{Cu}$ by alpha particle impact. For computations we used the expression $\bar{r}=\frac{R}{n_{e}^{1 / 3}}$ for mean distance $(\bar{r})$, where $R$ is radius of particular shell of the target atom. The factor $\frac{n_{e}\left(n_{e}-1\right)}{4 \pi \bar{r}^{2}}$ has been suitably modified to include the mode of ionization in which the electrons are ejected from different shells. Here $n_{e}$ is number of electrons in the shell under consideration, $Z$ is the charge on the projectile (for proton and electron $Z=1$ and 2 for alpha particle); $n_{e}\left(n_{e}-1\right)$ has been replaced by $n_{e 1} \times n_{e 2}$ where $n_{e 1}$ and $n_{e 2}$ stand for number of electrons in the shells under consideration. The values of $u_{i}$ and $u_{i i}$ stand for first and second ionization potentials and necessary quantum mechanical values of orbital energy and radial distance of maximum probability from Clementi and Roetti [31] and Desclanx [32] respectively.

Finally, total direct double ionization cross section of $\mathrm{Cu}$ is,

$Q_{D}^{i i}=Q_{D}^{i i}(4 s, 3 d)+Q_{D}^{i i}(4 s, 3 p)$

Where $Q_{D}^{i i}(4 s, 3 d)$ and $Q_{D}^{i i}(4 s, 3 p)$ stand for the direct double ionization cross section (DDICS) corresponding to ejection of one electron from $4 \mathrm{~s}$ orbital and the other either from the $3 p$ orbitals or $3 \mathrm{~d}$ orbital respectively.

\section{RESULTS AND DISCUSSION}

The calculated results of DICS along with experimental observations [33] have been shown in Table 1 and Fig.1. In Fig.1 curves of (4s, 3d) and $(4 s, 3 p)$ stand for the contribution of direct double ionization cross sections to the total theoretical double ionization cross sections for the corresponding impact energy. The terms Total and Expt. represent total theoretical and total experimental DICS in the Table 1respectively. Major contributions to DICS of $\mathrm{Cu}$ have been recorded from the pair of orbitals $(4 \mathrm{~s}$, $3 d)$. The contributions from orbitals $(4 s, 3 p)$ to DICS are found insignificant in different impact energies.

In the lower energy range of $54 \mathrm{keV}$ to 75 $\mathrm{keV} / \mathrm{amu}$, the theoretical results overestimate the experimental data and the ratio factor is greater than 2. Our results come closer to experimental data of total double ionization cross sections with the increase of impact energy. The theoretical value of DICS at impact energy $300 \mathrm{keV} / \mathrm{amu}$ is $0.98 \times 10^{-16} \mathrm{~cm}^{2}$ which is same as experimental values at that impact energy. Energy above of 300 $\mathrm{keV} / \mathrm{amu}$, the calculated results underestimates the 
experimental data and at $360 \mathrm{keV} / \mathrm{amu}$ their magnitude are $0.77 \times 10^{-16} \mathrm{~cm}^{2}$ and $1.0 \times$ $10^{-16} \mathrm{~cm}^{2}$ respectively. Here contribution of $(4 \mathrm{~s}$, $3 p)$ has negligible contribution. It is because of the low energy state of electron in $3 \mathrm{p}$. Electrons in $4 \mathrm{~s}$ and $3 \mathrm{~d}$ orbitals are in high energy states and yield major contribution to the cross sections of double ionization. Energy above $75 \mathrm{keV} / \mathrm{amu}$, all the theoretical results have ratio factor within 2. Out of the total number of theoretical data $75 \%$ are under valid range of ratio factor 2 and among the valid range $41 \%$ have ratio factor below 1.325 and hence calculated results of DICS lie in the acceptable range.

Table 1: Double ionization cross sections of $\mathrm{Cu}$ atom by $\mathrm{He}^{2+}$ particle in the unit of $\times 10^{-16} \mathrm{~cm}^{2}$.

\begin{tabular}{|c|c|c|c|c|}
\hline \multirow[t]{2}{*}{ E(KeV/amu) } & \multicolumn{2}{|c|}{ Contribution of } & \multirow[t]{2}{*}{ Total } & \multirow[t]{2}{*}{ Expt.[33] } \\
\hline & $(4 s, 3 d)$ & $(4 s, 3 p)$ & & \\
\hline 54 & 3.04 & 0.04 & 3.08 & $1.10 \pm 0.40$ \\
\hline 62 & 2.91 & 0.04 & 2.95 & $0.90 \pm 0.30$ \\
\hline 75 & 2.70 & 0.04 & 2.74 & $1.30 \pm 0.20$ \\
\hline 88 & 2.51 & 0.04 & 2.55 & $1.30 \pm 0.20$ \\
\hline 105 & 2.29 & 0.04 & 2.33 & $1.20 \pm 0.20$ \\
\hline 125 & 2.06 & 0.04 & 2.10 & $1.20 \pm 0.10$ \\
\hline 150 & 1.81 & 0.03 & 1.84 & $1.00 \pm 0.10$ \\
\hline 180 & 1.56 & 0.03 & 1.59 & $1.20 \pm 0.10$ \\
\hline 213 & 1.34 & 0.03 & 1.37 & $1,04 \pm 0.09$ \\
\hline 250 & 1.14 & 0.03 & 1.17 & $0.95 \pm 0.07$ \\
\hline 300 & 0.96 & 0.02 & 0.98 & $0.98 \pm 0.07$ \\
\hline 360 & 0.75 & 0.02 & 0.77 & $1.00 \pm 0.08$ \\
\hline
\end{tabular}

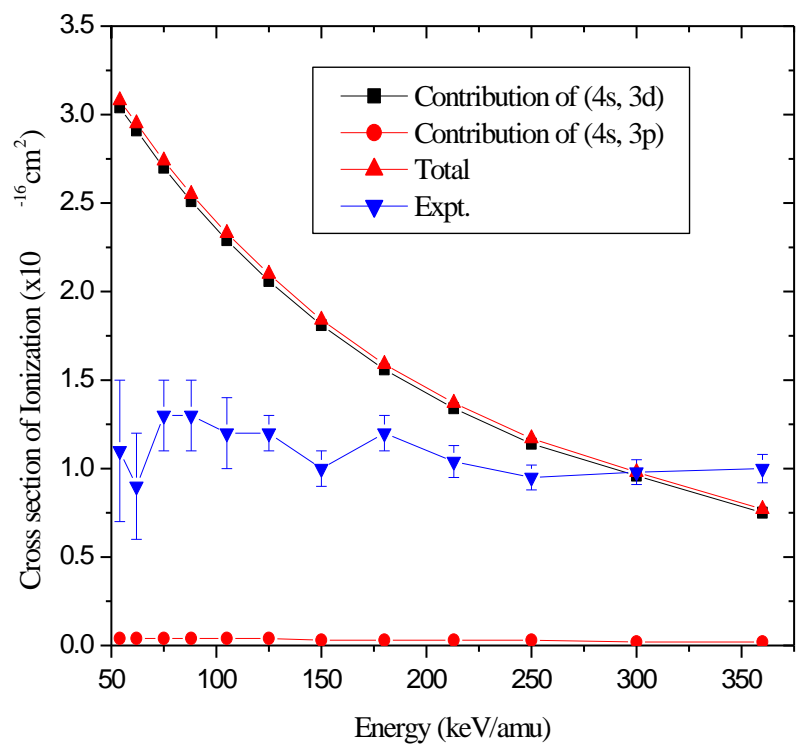

Fig. 1: $\mathrm{He}^{2+}$ impact DICS of $\mathrm{Cu}$ atom of in the given energy range. Here $\longrightarrow$ and $\longrightarrow-$ represents contribution to the cross section of ionization from pair of orbitals (4s, 3d) and (4s, 3p) and $\longrightarrow$ and represents Experimental and total theoretical values of DICS of $\mathrm{Cu}$.

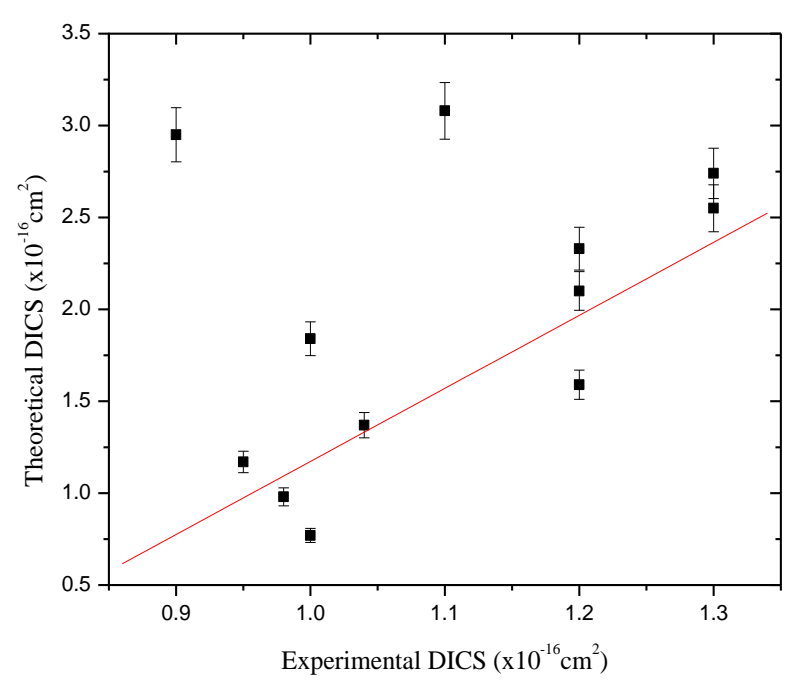

Fig. 2: Linear fit for theoretical results with experimental data. Error bars represent errors associated with theoretical values with respect to experimental data of DICS of $\mathrm{Cu}$.

The variation of error associated with theoretical results with the corresponding experimental values has been shown in Fig. 2. The magnitude of error 
bar increases with the increase of cross section of ionization. This revels that, in general, theoretical results possess more errors in threshold energy range where cross sections of ionization have higher values. The linear correlation coefficient ( $R$ square) and standard deviation (SD) of linear fit are 0.6984 and 0.7883 respectively. This shows that about $70 \%$ of theoretical results of DICS are in close agreement to the experimental data of DICS and smaller value of standard deviation certifies that the theoretical results have less uncertainty. In low energy range the theoretical results are more apart from corresponding experimental values and possess relatively more error compared to intermediate and higher energy region.

\section{CONCLUSION}

The computational calculations of double ionization cross sections of $\mathrm{Cu}$ by $\mathrm{He}^{2+}$ under semi-classical model of binary encounter approximation are in satisfactory agreement with the available experimental data. The results are explained by taking into account of contribution of two pair of orbitals $(4 s, 4 p)$ and $(4 s, 3 d)$. Here one electron is ejected from $4 \mathrm{~s}$ and next electron is either from $3 \mathrm{~d}$ or $3 p$ for double ionization. Normal $\mathrm{Cu}$ atom $\left[1 \mathrm{~s}^{2}\right.$ $2 s^{2} 2 p 63 s^{2}$ ] $3 p 63 d^{10} 4 s^{1}$. Here for double ionization of $\mathrm{Cu}, 4 \mathrm{~s}^{1}$ loses an electron first during the interaction with projectile ion and next one electron is from $3 \mathrm{~d}^{10}$ resulting the atom to state $\left[1 \mathrm{~s}^{2}\right.$ $\left.2 s^{2} 2 \mathrm{p} 6 \quad 3 \mathrm{~s}^{2}\right] \quad 3 \mathrm{p} 6 \quad 3 \mathrm{~d}^{9} 4 \mathrm{~s}^{0}$. This is not an auto ionization state and no further ionization happen. Possibility of double ionization is only by direct double ionization by alpha particle. Electrons of $3 p$ are at low energy state compared to $4 \mathrm{~s}$ and $3 \mathrm{~d}$ states. So ionization of electron from $3 p$ has less chance and hence values of DICS for $(4 s, 3 p)$ have very small values for given impact energies. Out of the total number of theoretical data $75 \%$ are under valid range of ratio factor 2 and among the valid range $41 \%$ have ratio factor below 1.325 and hence calculated results of DICS lie in the acceptable range. The model fails to account the physical insight of double ionization around threshold energy range. The energy imparted to the target atom spreads among orbital electron producing number of affects in threshold energy region and is of quantum nature which has not been included in our present semi classical mathematical formulation of binary encounter. The results can be improved by using density function theory (DFT). However, in general, majority of the calculated results are in satisfactory agreement with experiment.

\section{REFERENCES}

[1] Ehrhardt, H.; Jung, K.; Knoth, G.; and Schlemmer, P. Differential cross sections of direct single electron impact ionization. Zeitschrift für Physik D Atoms, Molecules and Clusters, 1(1): 3-32 (1986).

[2] Campbell, L., and Brunger, M. J. Electron collisions in atmospheres, International Reviews in Physical Chemistry, 35: 297-351 (2016).

[3] Lavvas, P.; Yelle, R.V.; Heays, A. N.; Campbell, L.; Brunger, M. J.; Galand, M. and Vuitton, V. $\mathrm{N}_{2}$ state population in Titan's atmosphere, Icarus, 2015 260, 29-59. 10.1016/j.icarus.2015.06.033

[4] Makabe, T. and Petrovic, Z. L. Plasma electronics: Applications in microelectronic device fabrication, 2nd edition, CRC Press, India, 2019.

[5] Bug, M. U.; Gargioni, E.; Nettelbeck, H.; Baek, W. Y.; Hilgers, G.; Rosenfeld, A. B. and Rabus, H. Ionization cross section data of nitrogen, methane, and propane for light ions and electrons and their suitability for use in track structure simulations, Physical Review E, 88(4): 043308043329 (2013).

[6] Hogstrom K. R. and Almond P. R. Review of electron beam therapy physics. Phys. Med. Biol., 51(13): R 455 (2006).

[7] Campbell, L.; Cartwright, D. C. and Brunger, M. J. Role of excited $\mathrm{N}^{2}$ in the production of nitric oxide. Journal of Geophysical Research: Space Physics, 112(A8): ..... (2007).

[8] Campbell, L. and Brunger, M. J. Electron impact contribution to infrared NO emissions in auroral conditions. Geophysical research letters, 34(22): .... (2007).

[9] Campbell, L. and Brunger, M. J. Modeling of kinetic, ionospheric and auroral contributions to the 557.7-nm nightglow. Geophysical research letters, 37(22): ....(2010).

[10] Egitto, F. D. Plasma etching and modification of organic polymers. Pure and applied Chemistry, 62(9): 1699-1708 (1990).

[11] Laroussi $\mathrm{M}$. and $\mathrm{Lu} \mathrm{X}$. Room-temperature atmospheric pressure plasma plume for biomedical applications. Appl. Phys., Lett. 87(11): 113902 (2005).

[12] Von Woedtke, T.; Reuter, S.; Masur, K. and Weltmann, K. D. Plasmas for medicine. Phys. Rep., 530(4): 291-320 (2013).

[13] Morfill, G. E.; Kong, M. G. and Zimmermann, J. L. Focus on plasma medicine. New Journal of Physics, 11(11): 115011 (2009).

[14] Pindzola, M. S.; Ludlow, J. A.; Robicheaux, F.; Colgan, J. and Griffin, D. C. Electron-impact double ionization of magnesium. J. Phys. B: At., Mol. Opt. Phys., 42(21): 215204 (2009). 
[15] Pindzola, M. S.; Ludlow, J. A.; Ballance, C. P.; Robicheaux, F. and Colgan, J. Electron-impact double ionization of $\mathrm{B}^{+}$. J. Phys. B: At., Mol. Opt. Phys., 44(10): 105202 (2011).

[16] Montanari C. C. and Miraglia J. E. Multiple ionization of argon atoms by helium - ions. J. Phys. B - At Mol. Opt, Phys., 49(17): 175203 175210 (2016).

[17] Gryziński, M. Classical theory of atomic collisions. I. Theory of inelastic collisions. Physical Review, 138(2A): A336 (1965).

[18] Chatterjee, S. N. and Roy, B. N. Electron impact double ionisation of $\mathrm{Ar} 2+, \mathrm{Ar} 3+$ and $\mathrm{Xe}+$. Journal of Physics B: Atomic and Molecular Physics, 20(10): 2291 (1987).

[19] Chatterjee, S. N. and Roy, B. N. Electron impact double ionisation of $\mathrm{Ar} 2+, \mathrm{Ar} 3+$ and $\mathrm{Xe}+$. Journal of Physics B: Atomic and Molecular Physics, 20(10): 2291 (1987).

[20] Jha, L. K. and Roy, B. N. Electron impact single and double ionization of magnesium. The European Physical Journal D-Atomic, Molecular, Optical and Plasma Physics, 20(1): 5-10 (2002).

[21] Jha, L. K.; Kumar, S. and Roy, B. N. Electron impact single and double ionization of argon. Eur Phys J D Atom Mol Opt Phys., 40(1): 101-106 (2006).

[22] Kumar, A. and Roy, B. N. Proton impact double ionisation of noble-gas atoms. J. Phys. B: At. Mol. Phys., 14(3): 501 (1981).

[23] Vriens, L. Binar - encounter proton-atom collision theory. Proceeding of the Physical Society, 90(4): 935-942 (1967).

[24] Kumar, A. and Roy, B. N. Application of the binary-encounter theory to proton impact double ionisation of atoms. J. Phys. B: At. Mol. Phys., 10(15): 3047 (1977).
[25] Gupta, S. P.; Jha, L. K and Khanal, R. Study of proton and alpha particle impact double ionization of $\mathrm{Fe}$ atom. Bulletin of Pure and Applied Science, D 36: 43-51 (2017).

[26] Singh, M. P.; Chatterjee, S.N.; Jha, L.K. and Roy, B.N. Single and double ionization of magnesium by $\mathrm{H}^{+}$and $\mathrm{He}^{2+}$ impact, Physica Scripta., 80(2): 25302-025400 (2009).

[27] Gryzinski, M. and Kunc, J. A. Double ionization of atoms by electrons. J. Phys. B- At. Mol. Opt. Phys., 32(24): $5789-5804$ (1999).

[28] Burgess, A. and Percival, I. C. Classical theory of atomic scattering (Chapter-5). Academic Press, New York. 1968.

[29] Younger, S. M. and Märk, T. D. Semi-empirical and semi-classical approximations for electron ionization. Electron impact ionization, Springer, Vienna, 24-41 (1985).

[30] McDowell, M. R. Classical impulse approximations, Proceedings of the Physical Society, 89(1): 23-27 (1966).

[31] Clementi E., and Roetti C. Roothaan-HartreeFock atomic wave functions: Basis functions and their coefficients for ground and certain excited states of neutral and ionized atoms, $\mathrm{Z} \leq 54$, Atomic Data and Nuclear Data Tables., 14(3-4): 177-478 (1974).

[32] Desclaux, J. Relativistic Dirac-Fock expectation values for atoms with $\mathrm{Z}=1$ to $\mathrm{Z}=120$ Atomic Data and Nuclear Data Tables., 12(4): 311-406 (1973).

[33] Patton, C. J.; Shah, M. B.; Bolorizadeh, M. A. Geddes, J. and Gilbody, H. B. Ionization in collisions of fast $\mathrm{H}^{+}$and $\mathrm{He}^{2+}$ ions with $\mathrm{Fe}$ and $\mathrm{Cu}$ atoms, J. Phys. B- At. Mol. Opt. Phys., 28(17): 3889- 3898 (1995). 